\title{
3 Der Einfluss von Digital Leadership auf Organisationen im Gesundheitswesen
}

Steffi Burkhart und Stephan Grabmeier

\section{Bestandsaufnahme und Stellenwert \\ Digitale Transformation im Gesundheitswesen}

Auch das Gesundheitswesen unterliegt dem Megatrend der Digitalisierung. Ob in privaten, kommunalen oder freigemeinnützigen Krankenhäusern, in Universitätskliniken, Pflegeeinrichtungen, Krankenkassen uvm. Im Rahmen der Gesamtstudie „Digitalisierungsindex Mittelstand“ der Deutschen Telekom in Zusammenarbeit mit techconsult, wurden auch Einrichtungen aus dem Gesundheitswesen gefragt, wie sie ihre Digitalisierungsbemühungen bewerten. Die Gesundheitsbranche erreicht 52 von 100 möglichen Index-Punkten und liegt damit im Durchschnitt aller Branchen (Deutsche Telekom AG 2016). 74 Prozent der medizinischen Einrichtungen befinden sich bereits auf dem Weg der digitalen Transformation.

Die digitale Transformation im Gesundheitswesen zielt darauf ab, die Innovationskraft zu steigern und Prozesse effizienter zu gestalten, um Patienten medizinisch besser zu versorgen und ihre Loyalität zu steigern.

Im Kontext E-Health (engl.: Electronic Health) geht es darum, elektronische Geräte, bei denen Informations- und Kommunikationstechnologie (IKT) bei der medizinischen Versorgung (Vorbeugung, Diagnose, Behandlung, Überwachung und Verwaltung) zum Einsatz kommen, zu implementieren (Albrecht 2016). Demgegenüber wird 
mHealth (engl.: Mobile Health) definiert als der Einsatz drahtloser Geräte wie Mobiltelefone, Phablets, Tablets, Patientenmonitore oder Personal Digital Assistants (PDA) bis hin zu Wearables und anderen tragbaren Geräte im Zusammenhang mit medizinischer Vorsorge und Versorgung (Kay et al. 2011; Elmer 2016). Mit mHealth Angeboten soll ein Paradigmenwechsel weg von rein empfangenen gesundheitlichen Modellen hin zu mitzugestaltenden Modellen, in denen Patienten an Selbstständigkeit (Empowerment) gewinnen, vollzogen werden (Albrecht 2016, 52).

Um als Organisation mit dem wachsenden Innovationsdruck von außen und der damit einhergehenden Veränderungsdynamik mithalten zu können, geht es nicht nur um die Implementierung neuer Technologien, sondern auch um eine Anpassung im Verhalten und im Organisationsdesign (siehe Kane et al. 2015).

\section{Digital Leadership}

Digitalisierung gehört als zentrales Thema in die Chefetage (Baculard 2017). Sie ist verantwortlich dafür, den digitalen Wandel erfolgreich zu gestalten und voranzutreiben. Aber was bedeutet „Digital Leadership“? CEOs müssen visionäre Leader sein, auch im Gesundheitswesen (Plsek u. Wilson 2001; Schwartz u. Tumblin 2002). Traditionelle, extrem hierarchisch geprägte Managementansätze greifen in einem dynamischen Umfeld ins Leere. Ein Mangel an guter Führung im Gesundheitssystem hat die Innovationskraft limitiert, die nötig ist, um kreative Lösungen für neue und komplexe Herausforderungen der Zukunft meistern zu können (Marquis u. Huston 2009).

\section{Digital Leadership fordert ein Mehr an wirklicher Führung, statt reinem Management - es bedarf einem ambidextrem (beidhändigem) Füh- rungsverständnis bestehend aus Management Skills und einem Lea- dership-Mindset (vgl. Tab. 1).}

\section{Tab. 1 Management versus Führung (Kotter 2015)}

Management $\quad$ Führung

Ambidextre Führung

(vgl. Ambidextrie im Organisationsdesign und der Mitarbeiterrolle in Abbildung 2)

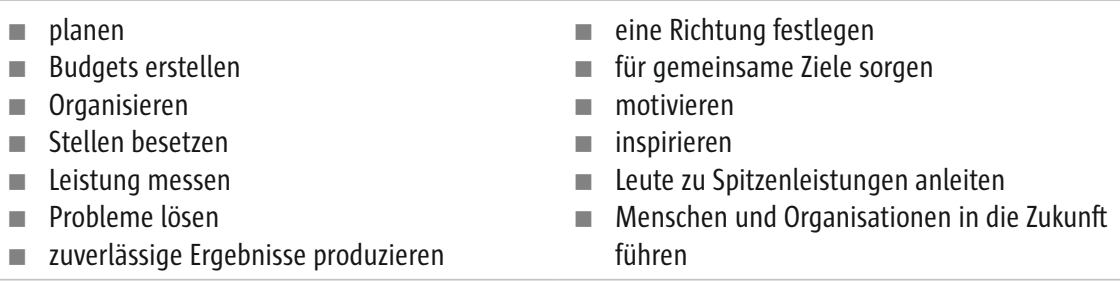


Denn mit einer rein managementgesteuerten Hierarchie und top-down Anweisungen über Silos hinweg, lässt sich eine so disruptive Veränderung, wie es sie die Digitale Transformation einer Organisation darstellt, nicht erfolgreich realisieren (Wilson et al. 2004).

Digital Leadership ist aber nicht nur eine Aufgabe der obersten Entscheider-Ebene, sondern durchzieht als „digital DNA“, die gesamte Organisation. Dazu gehört, Mitarbeiter auf allen Stufe der Organisation zu befähigen, gemeinsam Initiativen zu entwickeln und zu realisieren, eine Kultur des Experimentierens zu fördern, Kollaboration über die gesamte Organisation hinweg zu ermöglichen, eine auf Echtzeit basierende Informations- und Kommunikationsarchitektur zu implementieren und die Geschwindigkeit des Handelns zu erhöhen (Bersin 2016). Laut Kelley und Caplan besteht in der Ökonomie des 21. Jahrhunderts eine zentrale Aufgabe von Organisationen darin, die Produktivität der Wissensarbeiter zu erhöhen. Denn sie sind es, die letztendlich über den Erfolg eines Unternehmens entscheiden, nicht die Strategien und nicht die Technologie (Kelley u. Caplan 1993).

\section{Beschreibung des Transformationsprozesses}

Viele der weltweit führenden Change-Modelle basieren auf dem Ansatz des „Dualen Betriebssystems“ nach Kotter: Hierarchie versus Netzwerk (Ebene: Organisationsdesign), Change-Management versus Change-Leadership (Ebene: Mensch) (Kotter 1994; Übertragung auf das Gesundheitswesen: Campbell 2008). Dieser ist charakterisiert durch eine Anpassung auf der Verhaltensebene und im Organisationsdesign.

In dem Paradigmenwechsel zur Digitalisierung entsteht Erfolg nur durch Vielfalt und Vernetzung, durch ein Zusammenspiel von Organisationsdesign, Mitarbeiterbefähigung sowie Technologie (vgl. Abb. 1, Haufe, Holistischer Drei-Klang), was sich in einem Change-Ansatz widerspiegeln muss. Und zwar zeitgleich, nicht in einem Rollout und sequenziell gedacht.

Nur dann können Wirkungszusammenhänge entstehen. Denn: Was nützt ein Digital Leadership Programm (Mitarbeiterbefähigung), wenn die Führungskräfte keine technologisch gut ausgestattete Infrastruktur haben (Technologie)? Und was nützt das beste Social Network (Technologie), wenn die Kultur von Angst, Intransparenz oder Anweisung und Kontrolle geprägt ist (Organisationsdesign)? Oder was nützen agile Teams mit Gestalter-Persönlichkeiten (Mitarbeiterbefähigung), wenn interne Prozesse daran hindern zu experimentieren (Organisationsdesign)? Nichts. Deshalb besteht eine zentrale Aufgabe von Digital Leadern darin, Systembrüche zu beseitigen, indem integrierte Lösungen geschaffen werden, die die Komplexität berücksichtigen, statt sie zu verhindern. Deshalb hat Stephan Grabmeier von der Haufe AC den digitalen Change-Ansatz basierend auf diesem holistischen Drei-Klang entwickelt: 


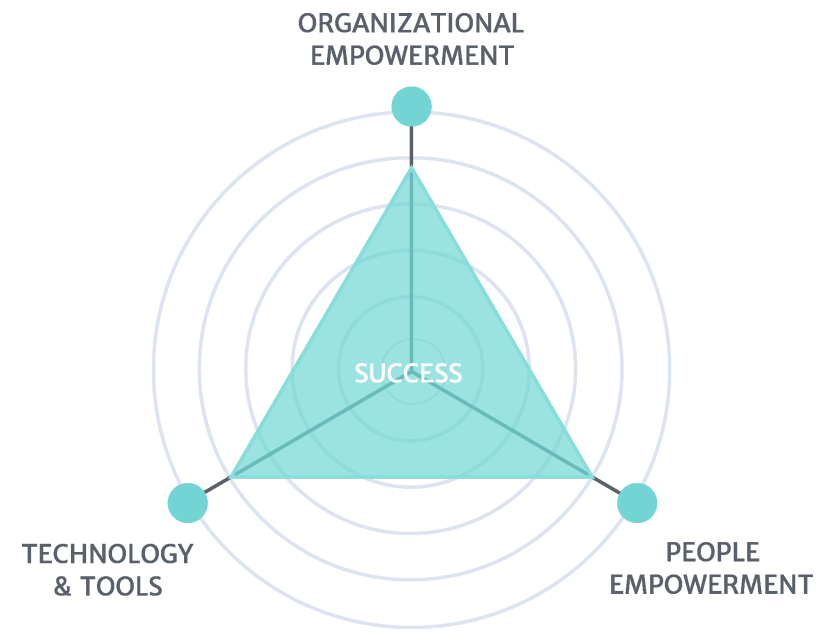

Abb. 1 Integration der drei Kernfaktoren: Organisation, Mitarbeiter und Technologie (Haufe Gruppe)

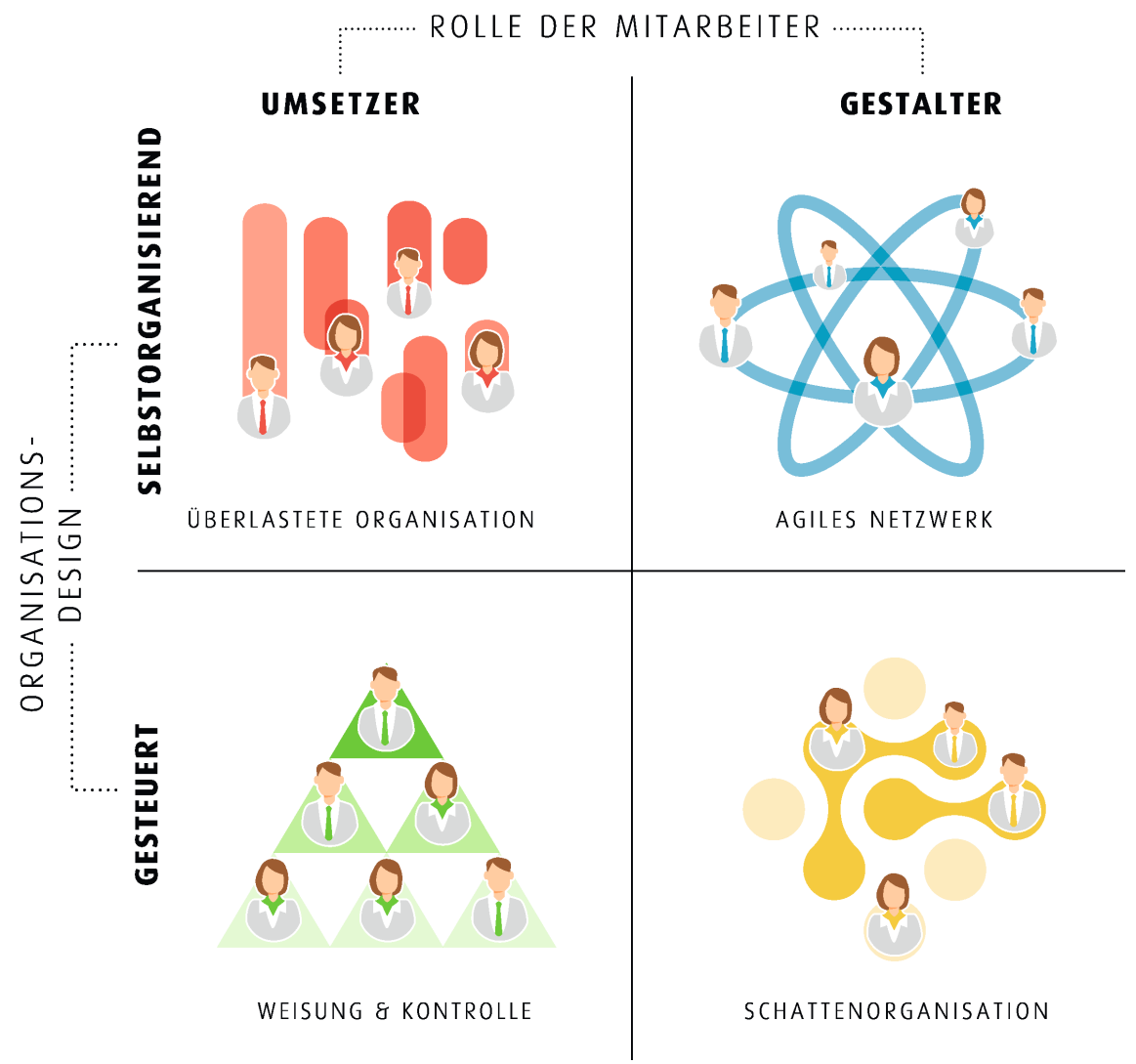

Abb. 2 Führung anpassen - Organisationsdesign und Mitarbeiterbefähigung (Haufe Gruppe) 


\section{Technologie \& Tools}

Die Einführung einer technologisch durchgängigen und mobilen Infrastruktur, basierend auf moderner Informations- und Kommunikationstechnologie (IKT) unterstützt dabei, alle Mitarbeiter, funktions- und hierarchieübergreifend auf einen gleichen Wissensstand zu bringen und zu halten. IKT allein löst jedoch noch keine Probleme und ändert keine Kultur.

\section{Mitarbeiterbefähigung}

Die Mitarbeiterbefähigung ist abhängig vom Führungsverständnis innerhalb einer Organisation sowie der Rolle, in der sich Mitarbeiter sehen. Haufe differenziert hierbei in ihrem Haufen-Quadranten (vgl. Abb. 2) zwischen der „Gestalter“- und „Umsetzer“-Rolle. Das Ausleben der jeweiligen Rolle ist abhängig vom Organisationsdesign.

\section{Organisationsdesign}

Der Haufe-Quadrant differenziert zwischen den beiden Polen starrer Steuerung, die auf effiziente Standardprozesse ausgerichtet ist und hoher Selbstorganisation, die auf Flexibilität und Innovation ausgerichtet ist.

\section{Chancen und Risiken}

Die Einführung einer „Digital Leadership-DNA“ und der damit einhergehenden Veränderung auf den Ebenen: Technologie, Verhalten und Organisationsdesign hat weitreichende positive Auswirkungen. Eine digitale DNA bringt eine Kultur hervor, die die Zusammenarbeit über Abteilungsgrenzen hinweg fördert und damit das SiloDenken und hierarchische Glaswände, die im Gesundheitswesen nach wie vor besonders stark ausgeprägt sind (Huston 2008), überwindet. Nach Schwartz und Tumblin ist die neue Führungskultur am erfolgreichsten, wenn es gelingt, Menschen in einer Organisation zu energetisieren. Darüber hinaus führt sie zu einem Wandel hin zu einer lernenden Organisation, in der traditionelle institutionelle Hierarchien abgeflacht sind und in welchen Veränderungen typischerweise team- und missionsorientiert stattfinden (Schwartz u. Tumblin 2002). In Kombination hat dies zur Folge, dass die Organisation an Dynamik und Agilität gewinnt. Ideen können schneller gemeinsam evaluiert und getestet, und neue Lösungen schneller gefunden werden. Die Organisation wird befähigt, auf den schnellen Wandel und das Chaos, das im Gesundheitswesen eingetroffen ist, zu reagieren. Eine solche Kultur kann eine qualitativ hochwertige Gesundheitsfürsorge sowie Sicherheit für Patienten und Mitarbeiter hervorbringen und gewährleisten (Huston 2008), was in Anbetracht der Masse an medizinischen Behandlungsfehlern, die nach wie vor im Gesundheitswesen vorherrschen, dringend notwendig ist.

Haufe hat im Gesundheitswesen eine große Krankenversicherung dabei unterstützt, sich in einigen Geschäftsbereichen digital zu transformieren. Dabei konnten unterschiedliche Risiken und Chancen eines Transformationsprojektes beobachtet werden: 


\section{Chancen}

- Sich als Unternehmen besser auf neue Kundenbedürfnisse und veränderte Ansprüche bezüglich digitaler Interaktion und Kommunikation (Social Media, Chat-Funktion etc.) einstellen können.

- Die Abwicklung von administrativen Dingen so einfach wie möglich gestalten. Bspw. das Einscannen von Belegen, digitale Unterschriften oder OneKlick Lösungen zur Kostenfreigabe.

- Schnelle Übermittlung von Daten zwischen Ärzten und Versicherung in der Abwicklung von Patienten-/Kundendaten, wodurch sich Bearbeitungszeiten erheblich reduzieren lassen.

\section{Risiken}

- Ablehnung von Mitarbeitern gegenüber moderner Technologie, wodurch Innovationen blockiert und Kunden vergrault werden.

- Kunden erwarten einen sicheren Umgang mit ihren Daten. Fehler in der Datensicherung führen zu einem Vertrauensverlust auf der Kundenseite. Umso wichtiger ist es, in die Handhabung der Datensicherung viele Ressourcen (Zeit, Geld, Know-how) zu investieren.

- Flache Hierarchien und modernes Leadership sind zentrale Voraussetzungen, um Mitarbeitern in der Fläche mehr Entscheidungsspielraum zu ermöglichen. Erst dadurch entstehen flexible Vorteile für Kunden. Identifizieren Sie mögliche Bremsklötze Ihrer Organisation, die Sie daran hindern, diese Voraussetzungen zu realisieren.

Nach zwei Jahren Projektbegleitung, mit über 13 Einzelinitiativen (z.B. Scannen von Belegen und einfache Kostenfreigabe, Digitale Antragsausfüllung bis zur Unterschrift, multioptionale Kommunikation mit der Versicherung über E-Mail, Telefon, Fax, Facebook oder WhatsApp sowie mobile Tarifrechner) lässt sich beobachten, dass Verharrungskräfte in der Versicherung und klassische Muster der Mitarbeiter und Führungskräfte den digitalen Change massiv beeinflussten.

\section{Ein erfolgreicher Change lässt sich nur realisieren, wenn eine gelebte top-down Unterstützung stattfindet und ein echter Wille zum Wandel vorliegt.}

\section{Trends und Entwicklung}

Auch wenn sich, wie eingangs beschrieben, bereits 74 Prozent der medizinischen Einrichtungen auf dem Weg der digitalen Transformation befinden, liegt deren digitaler Reifegrad - laut dem Global Healthcare Survey von Capgemini Consulting (Capgemini Consulting 2013), noch ziemlich im Anfangsstadium. Andererseits ist zu beobachten, dass immer mehr Konsumenten/Patienten digital Gesundheits-dienstleistungen und mHealth Angebote abrufen und einfordern. Diese Entwicklung treibt 
führende Technologieunternehmen wie Samsung, Apple oder Google dazu an, sich in den digitalen Healthcare-Sektor auszubreiten. Umso größer ist der Druck auf traditionelle Institutionen, sich schnell genug den veränderten Anforderungen von Konsumenten/Patienten und Treibern wie der Digitalisierung und dem Wunsch nach Individualisierung (personalized Health) und Konnektivität (connected Health) (Burkhart u. Hanser 2017), anzupassen. Das ist Aufgabe der Chefetage, die Dringlichkeit für einen digitalen Change aufzuzeigen, eine digitale Vision zu entwickeln und den Wandel erfolgreich zu führen.

\section{Literatur}

Albrecht UV (2016) Chancen und Risiken von Gesundheits-Apps (CHARISMHA). Medizinische Hochschule Hannover

Baculard LP (2017) To lead a digital transformation, CEOs must prioritize. Harvard Business Review

Bersin I (2016) Digital Leadership is not an optional part of being a CEO. Harvard Business Review.

Burkhart S, Hanser F (2017) Einfluss globaler Megatrends auf das digitale Betriebliche Gesundheitsmanagement (BGM). Digital Natives und Zukunftstrends als Treiber und Co-Creator für ein digitales, personalisiertes und vernetztes BGM., In: Matusiewicz, D. \& Kaiser, L. (Hrsg.) Digitales Betriebliches Gesundheitsmanagement. Theorie und Praxis. Wiesbaden: Springer Gabler (FOM-Edition)

Campbell RJ (2008) Change Management in Health Care. The Health Care Manager, 27(1), S. 23-39

Capgemini Consulting (2013) Global Healthcare Survey; Global Digital Maturity Assessment Survey by Capgemini Consulting and MIT Center for Digital Business

Deutsche Telekom AG (2016) Digitalisierungsindex. Der digitale Status Quo im Gesundheitswesen. Studie von techconsult im Auftrag der Deutschen Telekom AG

Elmer A (2016) eHealth in Deutschland - Probleme, Projekte, Perspektiven. Magazin des Wissenschaftlichen Instituts der A0K, 16(3), S. 7-13. URL: http://www.wido.de/fileadmin/wido/downloads/pdf_ggw/wido_ ggw_0316_elmer_0816.pdf. (zugegriffen am 28.07.2017)

Grabmeier S (2017) Rhythmix - Move your Company to a new Beat. URL: http://stephangrabmeier.de/rhythmix-move-your-company-to-a-new-beat/1340/ (abgerufen am 19.06.2017)

Huston C (2008) Preparing nurse leaders for 2020. Journal of Nursing Management, 16, S. 905-911

Kane GC, Palmer D, Phillips AN, Kiron D, Buckley N (2015) Strategy, not Technology, drives digital Transformation. MITSloan Management Review and Deloitte University Press

Kay M, Santos I, Takane M (2011) mHealth: New horizons for health through mobile technologies. World Health Organization 64(7), S. 66-71

Kelley R, Caplan I (1993) How Bell Labs creates star performers. Harvard Business Review, 71, S. 128-139

Kotter JP (1994) Leading Change: Why Transformation Efforts Fail. Harvard Business Review Press

Kotter JP (2015) ACCELERATE. Strategische Herausforderungen schnell, agil und kreativ begegnen. München: Verlag Vahlen, S. 46

Marquis B, Huston C (2009) Leadership Roles and Management Functions in Nursing: Theory and Application, 6th edn. Lippincott, Williams, \& Wilkins, Philadelphia, PA

Plsek PE, Wilson T (2001) Complexity, leadership, and management in healthcare organisations. BM], 323, S. $746-749$

Rotzinger J, Stoffel M (2015) Gelebte Demokratie. Harvard Business manager, Juli 2015

Schwarzt RW, Tumblin TF (2002) The Power of Servant Leadership to Transform Health Care Organizations for the 21st-Century Economy. Arch Surg. 137, S. 1419-1427

Wilson III EJ, Goethals GR, Sorenson G, Burns I M (2004) Leadership in the digital age. Encyclopedia of leadership, S. 858-861 